\title{
Differences among clinical isolates of Pseudomonas aeruginosa in their capability of forming biofilms and their susceptibility to antibiotics
}

\author{
DIDIK WAHYUDI ${ }^{1,2, \bullet}$, ABU THOLIB AMAN ${ }^{3}$, NIKEN SATUTI NUR HANDAYANI ${ }^{1}$, \\ ENDANG SUTARININGSIH SOETARTO ${ }^{1, \vee v}$ \\ ${ }^{1}$ Faculty of Biology, Universitas Gadjah Mada. Jl. Teknika Selatan, Sekip Utara, Sleman 55281, Yogyakarta, Indonesia. \\ Tel.: +62- 274-580839, " ${ }^{*}$ email: annisah-endang@ugm.ac.id. \\ ${ }^{2}$ Departement of Medical Laboratory Technology, Sekolah Tinggi Ilmu Kesehatan Nasional. Jl. Yos Sudarso 334, Surakarta 57155, Indonesia. \\ Tel.: +62-271-5723399, `email: didikww@gmail.com \\ ${ }^{3}$ Faculty of Medicine, Universitas Gadjah Mada. Jl. Sekip Utara, Sleman 55281, Yogyakarta, Indonesia.
}

Manuscript received: 11 February 2019. Revision accepted: 29 April 2019.

\begin{abstract}
Wahyudi D, Aman AT, Handayani NSN, Soetarto ES. 2019. Differences among clinical isolates of Pseudomonas aeruginosa in their capability of forming biofilms and their susceptibility to antibiotics. Biodiversitas 20: 1450-1456. Pseudomonas aeruginosa is an important nosocomial pathogen capable of causing both acute and chronic infections. The individuals of this bacterium have differences in their capability of forming biofilms. Biofilm is a collection of bacterial cells attached to the tissue, coated by polysaccharides and extracellular matrix, enabling bacteria to become resistant to antibiotics, so infection is difficult to treat. The aim of this study was to know the differences among clinical isolates of Pseudomonas aeruginosa in their capability of forming biofilms, and their susceptibility to some antibiotics. The bacteria were isolated from various patient samples at Dr. Moewardi Hospital Surakarta, Indonesia, from August to December 2017. The isolates were purified using single cell colony technique. The bacterial identification and tests of susceptibility to many antibiotic were conducted using automatic equipment (vitex® 2). Test method of biofilm formation was done using the Tissue Culture Plate. The readings of results were monitored spectrophotometrically at a wavelength of $570 \mathrm{~nm}$ and repeated 8 times. DNA isolation was done using Qiagen DNeasy Blood and Tissue Kit, and gene identification (pelD and pslA) was done using PCR (Polymerase Chain Reaction) and visualization through electrophoresis. The results showed that of the 64 isolates of $P$. aeruginosa from blood, sputum, urine, ear middle, urine catheter, pleural fluid, pus, stool, aspirate, and cerebrospinal, $22 \%$ were low in forming biofilm, 50\% moderate, and $28 \%$ high. Isolates of $P$. aeruginosa were resistant to ampicillin, cefazolin, tigecycline, nirofurantoin, and cotrimoxazole, but sensitive to piperacillin, ceftazidime, cefepime, aztreonam, meropenem, amikacin, gentamicin, and ciprofloxacin. The genes of pelD and $p s l A$ were present in all $P$. aeruginosa isolates (low, moderate, dan high). In conclusion, $P$. aeruginosa clinical isolates had different capability of forming biofilms and susceptibility to antibiotics. Isolates having high ability to form biofilm were relatively more resistant to many antibiotics. They were most sensitive to amikacin and resistant to ampicillin. There was no difference in the presence of mop pelD and pslA among all isolates.
\end{abstract}

Keywords: Pseudomonas aeruginosa, biofilm, susceptibility to antibiotic.

\section{INTRODUCTION}

Pseudomonas aeruginosa is a gram-negative, rodshaped bacterium, a leading cause of nosocomial infections, and an opportunistic pathogen. This bacterium causes respiratory tract infection, urinary tract infection, burns, cystic fibrosis and others (Catterjee et al. 2016). Pseudomonas aeruginosa is able to form biofilms, thus making treatment difficult. The incidence of resistance to antibiotics, chronic infections, and bacterial reinfection in the body is often associated with the process of biofilm formation (Deligianni et al. 2010).

Biofilm is a collective of one or more types of microorganisms (bacteria) that can grow on many different surfaces, tissues, or other materials covered by a binding layer composed of polysaccharides or extracellular matrix of bacteria (O'toole 2000; Andersson 2009). The biofilm matrix generally consists of $97 \%$ water, $2-5 \%$ microbial cells, 3-6\% of extracellular polymer substances (EPS) and ions. Extracellular polymers generally consist of 40-95\% polysaccharides, $1-60 \%$ protein, $1-10 \%$ nucleic acid and $1-$
40\% lipid. The composition of this EPS varies, depending on the composition of the microbial consortium and environmental factors (Andersson 2009).

Pseudomonas aeruginosa in a biofilm has different character than it does outside the biofilm. There have been several physiological changes in metabolism and enzyme expression as well as stimulation of virulence factors when $P$. aeruginosa forms biofilms. This bacterium has the ability to form biofilms that vary widely among individuals: high, moderate, low and even unable to form biofilms (Deligiani et al. 2010).

Pseudomonas aeruginosa becomes more resistant when forming biofilms within the tissue. Bacteria in the biofilm will form an extracellular matrix that protects bacteria from nearby disturbances, including immune cell invasion, extreme environmental conditions, invasive compound attacks including antibiotics (Taylor et al. 2014). The main constituent of $P$. aeruginosa biofilm is exopolysaccharide. The formation of exopolysaccharide is influenced by nutrients, oxygen, $\mathrm{pH}$, temperature, humidity, characteristics of surface on which it is attached, nitric 
oxide, some chemicals (biosurfactants) produced, and inhibitors of exopolysaccharide formation (Sakuragi and Kolter 2007; Hostaca et al. 2010; Kim and Lee 2016).

The formation of a biofilm of $P$. aeruginosa is controlled by three complex systems, namely quorum sensing system, cyclic dimeric guanosine monophosphate (c-di GMP), and GacS / GacA and RetS / LadS systems (Wei and Luyan 2013 and Rasamiravaka et al. 2015). The complex regulation of biofilm formation involves multiple bacterial machineries, including the Quorum Sensing system and the two-component regulatory system, both of which interact mainly with EPS production (De Keivit 2009). QS is a cell-to-cell communication used by many bacteria to detect their population density by producing and perceiving diffusible signal molecules that coordinate virulence factor production, motility, and biofilm formation. The GacS/GacA system acts as a superregulator of the QS system and is involved in the production of multiple virulence factors as well as in biofilm formation. Polysaccharide production is dependent on the intracellular pool of c-di-GMP, a ubiquitous intracellular second messenger widely distributed in bacteria. In bacterial cells, c-di-GMP is generated from two molecules of guanosine triphosphate by diguanylate cyclases and broken down into 2-GMP by specific phosphodiesterases (Rasamiravaka et al. 2015).

Pseudomonas aeruginosa has a variety of physiological, biochemical and genetic characteristics. The ability to form biofilms of some clinical isolates needs to be investigated. The type of infection, the severity of the infection, the length of infection can affect the formation of biofilm P. aeruginosa (Deligiani et al. 2010). The aim of this study was to know the differences among clinical isolates of $P$. aeruginosa in their capability of forming biofilms and their susceptibility to some antibiotics.

\section{MATERIALS AND METHODS}

Pseudomonas aeruginosa was isolated from various patient samples at Dr. Moewardi Hospital Surakarta, Indonesia, from August 2017 to Mei 2018. The isolates were purified using single cell colony technique. The bacterial identification and tests of susceptibility to many antibiotics were conducted using automatic equipment (vitex ${ }^{\circledR} 2$ ). Preparation of $P$. aeruginosa cultures was done in BHI media, incubated at $37^{\circ} \mathrm{C}$ for 24 hours. Antibiotics used consisted of ampicillin, ampicillin-sulbactam, piperacillin, cefazolin, ceftazidime, cefepime, aztreonam, meropenem, amikacin, gentamicin, ciprofloxacin tigecycline, nitrofurantoin, and cotrimoxazole.

Table 1. Interpretation of biofilm production

\begin{tabular}{ll}
\hline Average OD value & Biofilm production \\
\hline$\leq$ ODc $/$ ODc $<\sim \leq 2 \mathrm{x}$ Odc & Non / Low \\
$2 \mathrm{x}$ ODc $<\sim \leq 4 \mathrm{x}$ Odc & Moderate \\
$>4 \mathrm{x}$ Odc & High \\
\hline Optical density cut-off value $(\mathrm{ODc})=$ average OD of negative \\
control $+3 \mathrm{x}$ standard deviation $(\mathrm{SD})$ of negative control
\end{tabular}

Biofilm formation test was done using Tissue Culture Plate method. Pseudomonas aeruginosa isolated from fresh agar plates were inoculated in $10 \mathrm{~mL}$ of trypticase soy broth with $1 \%$ glucose. Broths were incubated at $37^{\circ} \mathrm{C}$ for $24 \mathrm{~h}$. The cultures were then diluted 1:100 with fresh medium. Individual wells of 96 sterile wells with flat bottom polystyrene tissue culture plates were filled with $200 \mu \mathrm{L}$ of the diluted cultures. The control organisms were also incubated, diluted and added to tissue culture plates. Negative control wells contained inoculated sterile broth. The plates were incubated at $37^{\circ} \mathrm{C}$ for $24 \mathrm{~h}$. After incubation, contents of each well were removed by gently tapping it. The wells were washed with $0.2 \mathrm{~mL}$ of phosphate buffer saline ( $\mathrm{pH}$ 7.2) four times. This removed free-floating bacteria. The biofilms formed by bacteria adherent to the wells were fixed with $2 \%$ sodium acetate and stained with crystal violet $(0.1 \%)$. Excess stain was removed using deionized water and plates were kept for drying. The growth was monitored spectrophotometrically at a wavelength of $570 \mathrm{~nm}$, with 8 replications. The interpretation of biofilm production was done according to the criteria in Table 1 (Hassan et al. 2011).

DNA isolation was done using Qiagen DNeasy Blood and Tissue Kit to represent $P$. aeruginosa isolates, each with 10 isolates with low, moderate, and high abilities, then identification of the presence of the pelD and pslA genes (the two main genes that control $P$. aeruginosa biofilm formation) was done using a primer for the pelD gene forward: AAT CAT ATG AAC GAC CAG AGC CTG CGC AGT, reserve: TCC CTC GAG CTA AAC AGC CAC TTG CTG ATC (Vasseur et al. 2005), and primer for pslA gene forward: CGC ATC ATG TTC TTC GCC TG, reverse: GTA CAT GCC GCG TTT CAT CC (NCBI, 2018).

\section{RESULTS AND DISCUSSION}

The identification found 64 clinical isolates of $P$. aeruginosa, consisting of (3\% blood, $27 \%$ sputum, $9 \%$ urine $16 \%$ ear middle, $8 \%$ urine catheter, $6 \%$ pleural fluid, $23 \%$ pus, $2 \%$ stool, $5 \%$ aspirate, $2 \%$ cerebrospinal). Of the 64 isolates, $22 \%$ were found low in forming biofilms, $50 \%$ moderate, and $28 \%$ high (Figure 1 ).

The results showed that $P$. aeruginosa was able to infect several parts of the body. Pseudomonas aeruginosa was found in 10 types of samples, namely blood, sputum, middle ear urine, urine catheter, pleural fluid, pus, stool, aspirate, and cerebrospinal. The most common $P$. aeruginosa isolates were found in sputum, pus and ear samples, and a few samples of blood, stool, and cerebrospinal fluid. Pseudomonas aeruginosa is found to infect the respiratory and pulmonary tract (sputum), wounds, ear, and urinary tract. We found the different ability of $P$. aeruginosa isolates to form biofilms although they were from the same sample type. No patterns were found in the capability of forming biofilms among sample types; some isolates that had high biofilm formation ability were found in sputum samples and pus. (Figure 2). 


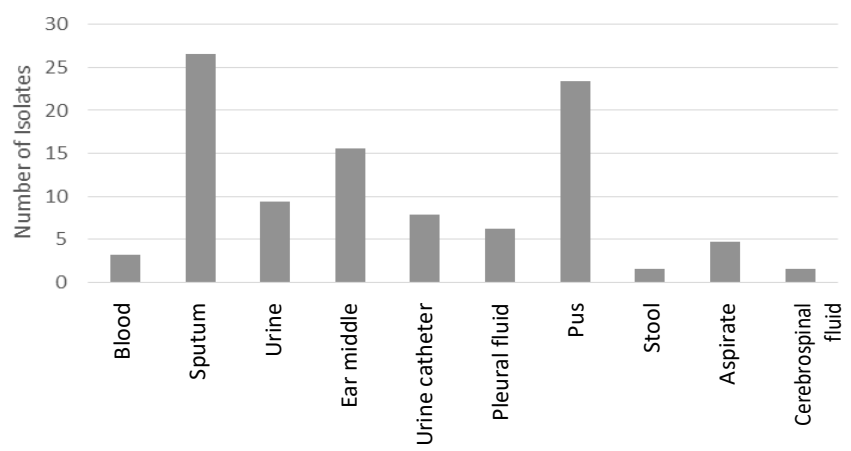

A

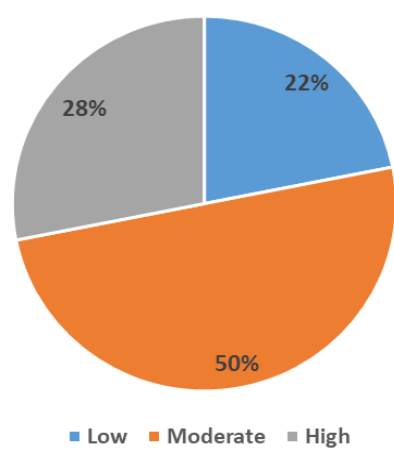

B

Figure 1. Clinical samples of Pseudomonas aeruginosa distribution (A), and biofilm formation (B).

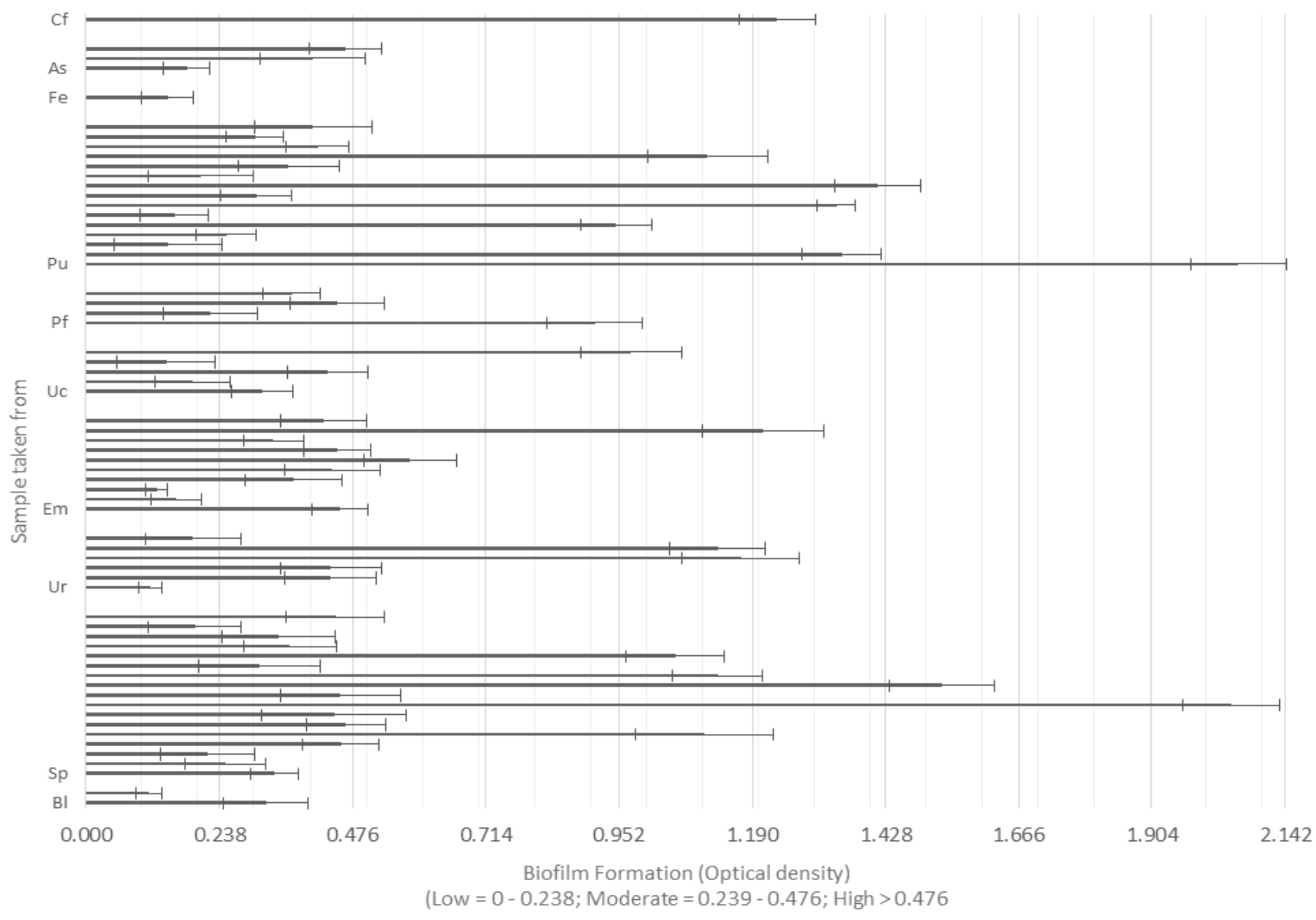

Figure 2. Biofilm Formation Pseudomonas aeruginosa from various types of clinical samples.Note: Bl: Blood, Sp: Sputum, Ur: Urine, Em: Ear middle, Uc: Urine catheter, Pf: Pleural fluid, Pu: Pus, Fe: Feces, As: Aspirate, Cf: Cerebrospinal fluid

Pseudomonas aeruginosa was resistant to ampicillin, cefazolin, tigecycline, nitrofurantoin and trimethoprimsulfamethoxazole (cotrimoxazole), but sensitive to piperacillin, ceftazidime, cefepime, aztreonam, meropenem, amikacin, gentamicin, and ciprofloxacin. In this study, amikacin was the most effective antibiotic against $P$. aeruginosa, while ampicillin was the least effective (Figure 3).

Groupings of the ability of biofilm formation and the susceptibility to 14 antibiotics show that isolates with high ability to form biofilms have a tendency to be more resistant to more antibiotics, compared with those having moderate and low ability (Figure 4).

The DNA of $P$. aeruginosa was isolated with Qiagen DNeasy Blood and Tissue Kit reagents. The results of DNA isolation for 30 Isolates of $P$. aeruginosa (each representative of 10 isolates for low, moderate, and high biofilm) were subsequently identified with pelD and pslA genes. Prior to this, optimization tests on primers had been made and designed to identify pelD and $p s l A$ genes. 


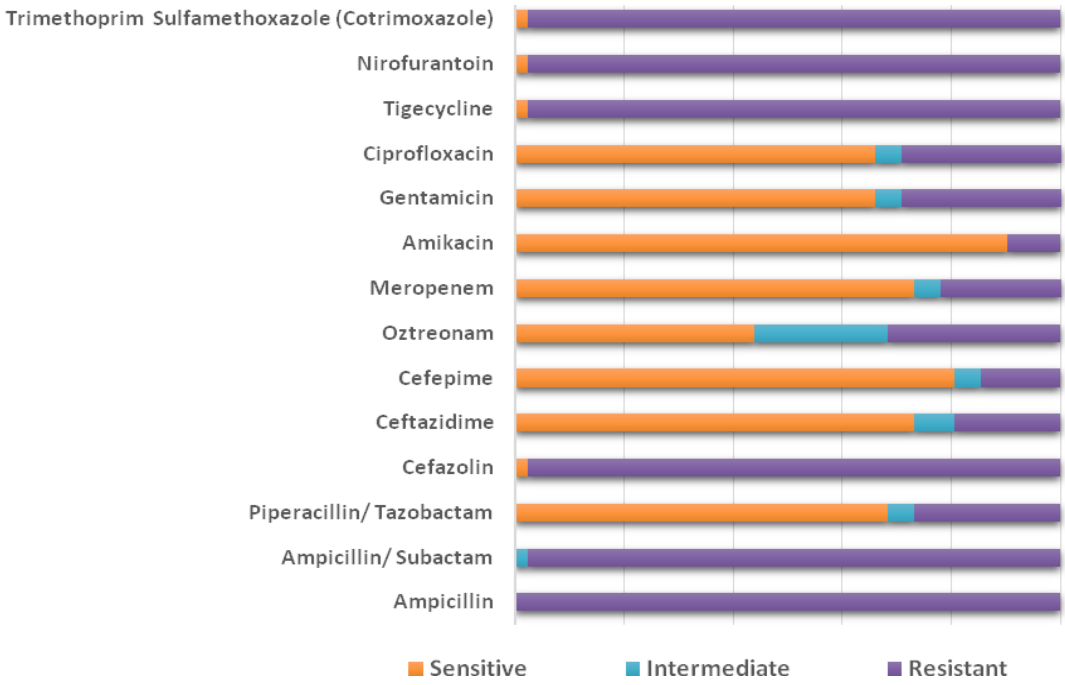

Figure 3. Susceptibility of Pseudomonas aeruginosa clinical isolates to different antibiotics

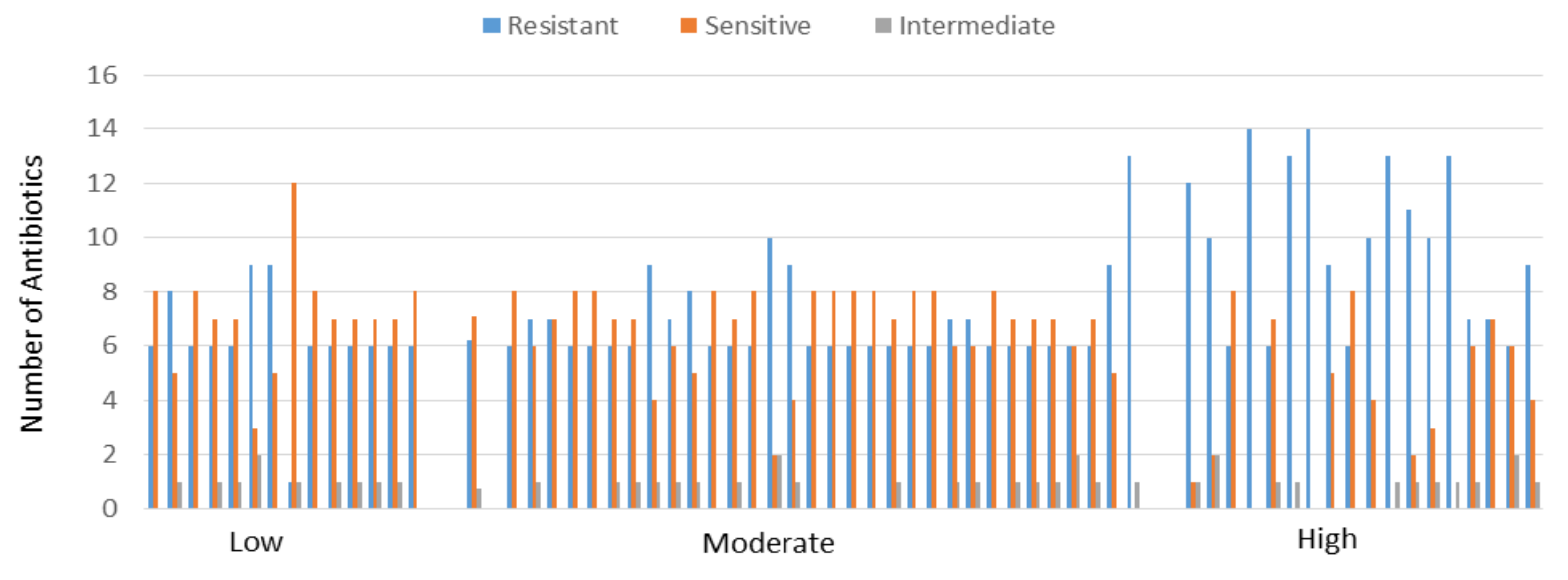

Biofilm Formation of $P$. aeruginosa

Figure 4. Biofilm formation and antibiotic susceptibility of Pseudomonas aeruginosa
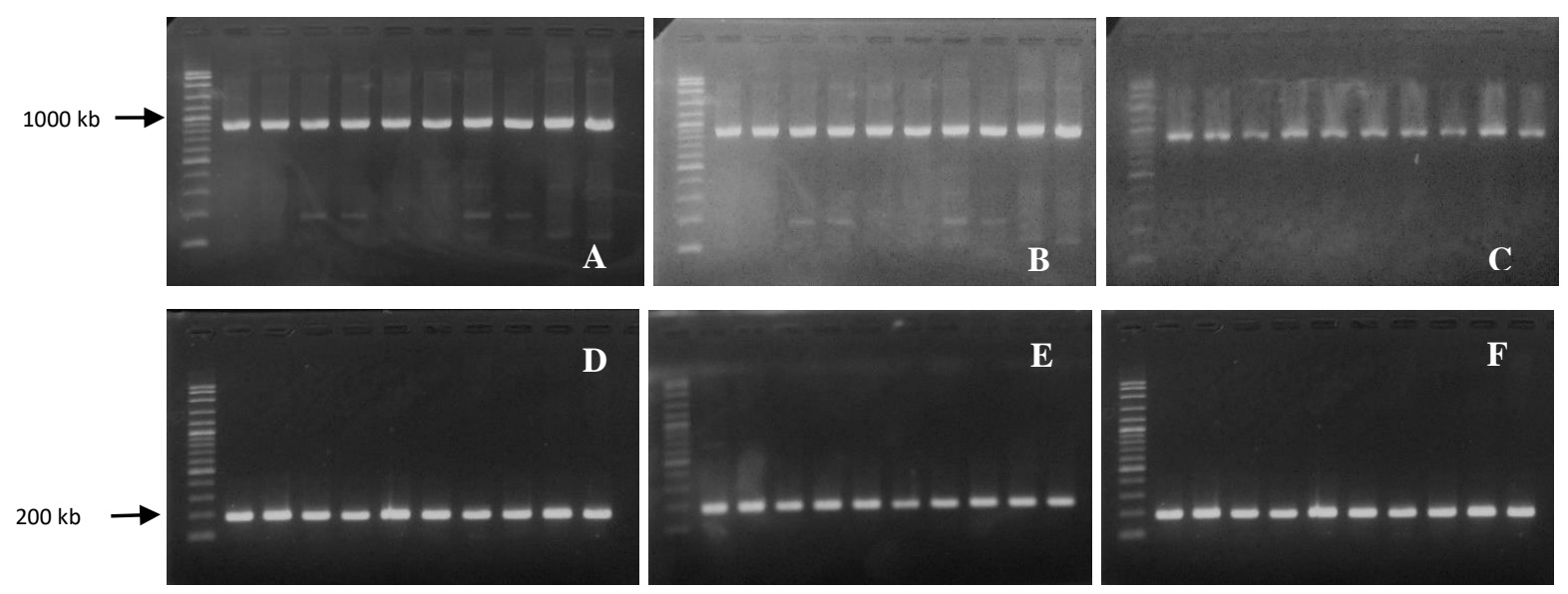

Figure 5. Results of visualization of pelD gene PCR products (above) and pslA (below), A and D isolates with low ability, B and E isolates with moderate ability, $\mathrm{C}$ and $\mathrm{F}$ isolates with high ability in biofilm synthesis 
The primary optimization test for pelD and pslA was carried out using the PCR program and continued with the visualization of PCR products using electrophoresis. Each gene was tested using primers namely the pelD gene. The results of visualization using electrophoresis are shown in Figure 5.

The results of primary pelD and $p s l A$ gene visualization showed that the pelD and $p s l A$ genes were found in all $P$. aeruginosa isolates, both those with low, moderate and high ability to form biofilms. pelD gene-specific primers have a PCR product of $887 \mathrm{~kb}$, and pslA gene primers of $189 \mathrm{~kb}$.

\section{Discussion}

The results showed that $P$. aeruginosa infects almost all parts of the body. Pseudomonas aeruginosa was found in blood, sputum, middle ear urine, urine catheter, pleural fluid, pus, stool, aspirate, and cerebrospinal. If it is associated with its ability to form biofilms, the formation of biofilms was not affected by the site of infection in the body (Figure 2). Some isolates obtained from the same type of sample showed differences, for example in sputum samples, 17 isolates had different capability of forming biofilms. Likewise in the pus sample, of the 15 isolates found, the ability to form biofilms varied, i.e., low, medium and high. The ability to form biofilms is influenced by genetic factor of $P$. aeruginosa, and other supporting factors affecting the formation of extracellular polymeric material for the preparation of $P$. aeruginosa biofilm matrix

Pseudomonas aeruginosa belongs to the group of proteobacteria, capable of degrading proteins and possessing exoprotease enzymes. The production of these exoproteases is controlled by the quorum sensing system, similar to the formation of biofilms. P. aeruginosa that infects organs removes virulence factors that cause cell and tissue damage. The expression of some enzymes and polysaccharides, because of these infections, leads to an increase in biofilm formation. $P$. aeruginosa will be higher if they form an exclusive community in biofilms: the more bacterial cells that join, the more nutrients will be available, and the more protected they are from the antibacterial and immune system that will destroy them.

Isolates of $P$. aeruginosa were different in their capability of forming biofilms. Most $P$. aeruginosa isolates had moderate ability to form biofilms. When correlated with antibiotic susceptibility, high isolates in forming biofilms were more resistant to many antibiotics than the low and moderate isolates. There was a significant difference in antibiotic sensitivity between the high and the low isolates, as well as between the high and the moderate isolates, but there was no significant difference between the low and the moderate isolates. P. aeruginosa which have high ability to form biofilms are more able to protect themselves from the influence of antibiotics, by forming layers of polysaccharides and thick exoprotease to defend themselves from exposure to antibiotics. Biofilms provide physical and chemical protection against antibiotic exposure.
There are 5 stages of biofilm formation on $P$. aeruginosa: (i) initial attachment, (ii) irreversible attachment, (iii) maturation I, (iv) maturation II, and (v) dispersion. Biofilm formation begins with the presence of organic and inorganic molecules dissolved in the tissue. The bacteria move and attach to the surface through Van der Waals, electrostatic, hydrogen, or Brownian bonds. Then, bacterial cells multiply and after reaching a certain number in the network, the bacteria secrete a signal molecule (autoinducer) called quorum sensing signal (acylhomoserine lactone signal), a chemical that connects bacterial cells (Wie and Luyan 2013), so this molecule is used by bacteria to communicate with each other. Furthermore, EPS production will strengthen the attachment of bacterial cells to the surface. The bonds between bacterial cells in the biofilms are irreversible (Periasamy et al. 2015), and result in the growth and development of the biofilm community. The higher the number of bacteria attached, the higher the number of signal molecules, and after reaching an adequate amount, the bacteria will express its virulence factor (Dieppois et al. 2009).

There were differences in the susceptibility to antibiotics among clinical isolates of $P$. aeruginosa. Piperacillin, ceftazidime, cefepime, aztreonam, meropenem, amikacin, gentamicin, and ciprofloxacin were more able to inhibit growth, than ampicillin, cefazolin, tigecycline, ole. Some plausible reasons for this are: (i). $P$. aeruginosa has intrinsic resistance to some antibiotics, (ii) frequently used antibiotics in the clinic will tend to be less effective, and vice versa. (iii) the dose of antibiotics affects the resistance of $P$. aeruginosa.

The ability to form biofilms and susceptibility to antibiotics in $P$. aeruginosa should be taken into consideration in the treatment of infectious diseases. Many researchers in some developed countries have examined the biofilm's contribution to the severity of a disease, and trying to find a strategy to inhibit the formation of bacterial biofilms.

Pseudomonas aeruginosa is an opportunistic pathogen, which will express its virulence factor when it has reached a certain concentration in tissue. Bacteria express a virulence factor along with changes in bacterial gene activity. There are different genes involved in this process and there are variations among individuals (Chugani and Greenberg 2010), leading to a variation in the ability to form biofilms among $P$. aeruginosa in this study. The involvement of the gene is related to the components of the polysaccharides or carbohydrates produced in the biofilm matrix. There are two genes that play a role in the production of carbohydrate components of biofilm matrix, namely pel genes and psl. pel gene plays a role in the production of glucose-rich material, while the psl gene contributes to the formation of mannose-rich biofilm matrix formed by most strains of $P$. aeruginosa (Wei and Luyan 2013).

In general, there was no variation in the presence of pel from the existing isolates, so the presence of this gene could not be used as a high indicator of low biofilm 
synthesis, especially in $P$. aeruginosa. However, further variations can be seen by sequencing the genes to see the arrangement of nitrogen bases or amino acids based on each isolate. This also occurs in the psl gene. The pslA gene is a gene that triggers the control of extracellular polymers which are constituent components of biofilms in $P$. aeruginosa. $p s l$ works together with other genes in forming $P$. aeruginosa biofilms.

The biofilm formation system in $P$. aeruginosa is controlled by three systems, namely the quorum sensing system, the setting of GacS / GacA and retS / Lad, and biosynthesis through c-in GMP (Rasamiravaka et al., 2015). The quorum sensing system will detect the number of bacteria by using a signal molecule (autoinducer), then stimulates the production of virulence factors, motility, and biofilm formation. $P$. aeruginosa has two main quorum sensing systems, namely las system and $r h l$ system, which encourage the production of all LasI and RhlI synthases. In other parts the quorum sensing system runs on a signal quinolone (PQS system), interacting with homoserine lactone acyl (AHLs) in a complicated manner (Jimenez et al. 2012). According to Gilbert et al. (2009), the lasor quorum sensing regulator can bind to the promoter region of the psl operon, indicating that quorum sensing can regulate psl expression.

The $r h l$ system plays a role in the formation of $P$. aeruginosa biofilms by increasing the biosynthesis of Pel polysaccharides. PQS plays a role in Edna formation during biofilm development. The quorum sensing system is also known to control motility, production of rhamnolipids and lectins. The motility is regulated by the rhl system. Flagella has a contribution in the early stages of $P$. aeruginosa formation related to bonding between bacterial cells. The production of rhamnolipid controlled by the $r h l$ system also has a role in $P$. aeruginosa biofilm formation, namely by forming microcolony and facilitating the formation of fungal-shaped structures on biofilms of $P$. aeruginosa, and facilitating the dispersion of bacterial cells (Dusane et al. 2010). Galactophilic lectin is a virulence factor (Leca and LecB) which is regulated by the quorum sensing $r h l$ system which has a role in the early stages of $P$. aeruginosa biofilm formation (Winzer et al. 2000).

The GacS / GacA system is a super-regulator of the quorum sensing system and is involved in the production of several virulence factors as well as in biofilm formation. The Gac system consists of a transmembrane kinase sensor $(G a c S)$ after autophosphorylation. The transfer of the phosphate group to its cognate regulator (GacA) plays a role in regulating RNA expression (RsmZ and RsmY). RsmZ and RSMY capture the RNA-binding protein RsmA regulator (encoded by the rsmA gene). A posttranscriptional repressor controls the psi locus (psa-L). At a later stage, the psl gene regulates the production of Psl polysaccharides, together with Pel polysaccharides, and alginate forms biofilm formation on P. aeruginosa (Irie et al. 2010).

Biofilms in everyday life have a positive and negative impact. The positive impact is that bacteria can decompose some compounds that are dangerous, toxic, and cause pollution in water, while the negative impact is that the contamination of water, food, and drink, contamination of medical equipment, catheters, implantation organs, pacemaker equipment can be a means of bacteria to colonize and can cause chronic infection which in turns worsens our health. Many researchers in several developed countries who have explored the contribution of biofilms to the severity of a disease, found a trigger factor and some of the effects that can be caused by the formation of bacterial biofilms in the equipment and the body.

In conclusion, $P$. aeruginosa clinical isolates had different capability of forming biofilms and susceptibility to antibiotics. Isolates which had high capability of forming biofilms were relatively more resistant to many antibiotics. $P$. aeruginosa clinical isolates were most sensitive to amikacin and resistant to ampicillin. There was no difference in the presence of gene pelD and pslA among all isolates.

\section{ACKNOWLEDGEMENTS}

We are grateful to the Ministry of Research, Technology and Higher Education, Republic of Indonesia, for doctoral dissertation grants, Decree Number 0045 / E3 / LL / 2018 and Agreement / Contract Number 092 / K6 / KM / SP2H / PENELITIAN / 2018.

\section{REFERENCES}

Andersson S. 2009. Characterization of Bacterial Biofilm From Wastewater Treatment. Royal Institute of Technology, School of Biotechnology, Stockholm, Sweden.

Chatterjee M, Anju CP, Biswas, Kumar VA, Mohan CG, Biswas R, 2016. Antibiotic resistance in Pseudomonas aeruginosa and alternative therapeutic options. Intl J Med Microbiol 306: 48-58.

Chugani S, Greenberg EP. 2010, LuxR homolog independent gene regulation by acyl-homoserine lactones in Pseudomonas aeruginosa. Proc Natl Acad Sci USA 107: 10673-10678.

Deligianni E, Pattison S, Berrar D, Ternan NG, Haylock RW, Moore JE, Elborn SJ, Dooley JS. 2010, Pseudomonas aeruginosa Cystic Fibrosis isolates of similar RAPD genotype exhibit diversity in biofilm forming ability in vitro. BMC Microbiol 10: 38. DOI: 10.1186/14712180-10-38.

De Kievit. 2009. Quorum sensing in Pseudomonas aeruginosa biofilms. Environ Microbiol 11 (2): 279-288.

Dieppois G1, Ducret V, Caille O, Perron K. 2012. The transcriptional regulator $\mathrm{CzcR}$ modulates antibiotic resistance and quorum sensing inPseudomonas aeruginosa. PloS One 7 (5): e38148. DOI: 10.1371/journal.pone.0038148.

Dusane DH, Zinjarde SS, Venugopalan VP, Mclean RJC, Weber MM, Rahman PKSM. 2010. Quorum sensing: implications on Rhamnolipid biosurfactant production. Biotechnol Genet Eng Rev 27: 159-184.

Gilbert KTHK, Gupta R, Greenberg EP, Schuster M. 2009, Global position analysis of the Pseudomonas aeruginosa quorum-sensing transcription factor LasR. Mol Microbiol 73, 1072-1085.

Hassan A, Javaid U, Fatima K, Maria O, Ali K, Muhammad I. 2011. Evaluation of different detection methods of biofilm formation in the clinical isolates. Department of Microbiology National University of Sciences and Technology, Islamabad, Army Medical College, Rawalpindi, Pakistan.

Hostacka A, Cizinar I, Stefkovcova M. 2010. Temperature and pH affect the production of bacterial biofilm. Folia Microb 55 (1) 75-78.

Irie YM, Starkey AN, Wozniak EJD, Romeo T, Parsek MR. 2010. Pseudomonas aeruginosa biofilm matrix polysaccharide Psl is regulated transcriptionally by $\mathrm{RpoS}$ and post-transcriptionally by RsmA. Mol Microbiol 78, no. 1: 158-172, 2010. 
Jimenez PN, Koch G, Thompson JA, Xavier KB, Cool RH, Quax WJ. 2012. The multiple signaling systems regulating virulence in Pseudomonas aeruginosa. Microbiol Mol Biol Rev 76, no. 1: 46-65.

Kim SK, Joon-Hee L. 2016. Biofilm dispersion in Pseudomonas aeruginosa. J Microb 54, No. 2: 71-85.

O'toole G, Kaplan HB, Kolter R. 2000. Biofilm formation as microbial development. Annu Rev Microbiol 54: 49-79.

Periasamy S, Nair HA, Lee KW, Ong J, Goh JQ, Kjellebergand S, Rice SA. 2015. Pseudomonas aeruginosa PAO1 exopolysaccharides are important for mixed species biofilm community development and stress tolerance. Original research. published: 20 August 2015.

Rasamiravaka T, Quentin L, Pierre D, Mondher El J. 2015, The formation of biofilms by Pseudomonas aeruginosa: A Review of the natural and synthetic compounds interfering with control mechanisms. Hindawi Publishing Corporation BioMed Res Int Volume-17.
Sakuragi Y, Kolter R. 2007. Quorum-sensing regulation of the biofilm matrix genes (pel) of Pseudomonas aeruginosa. J Bacteriol 189 (14): 5383-5386.

Taylor P, Amy TYY, Robert EWH. 2014. Antibiotic resistance in Pseudomonas aeruginosa biofilms: Towards the development of novel anti-biofilm therapies. J Bacteriol 191; 131-140.

Vasseur P, Isabelle VG, Chantal S, Stephane G, Alain F. 2005. The pel genes of the Pseudomonas aeruginosa PAK strain are involved at early and late stages of biofilm formation. Microbiology 151, 985997. DOI: 10.1099/mic.0.27410-0.

Wei Q, Luyan ZM. 2013. Biofilm matrix and its regulation in Pseudomonas aeruginosa. Int J Mol Sci 14, 20983-21005.

Winzer K, Falconer C, Garber NC, Diggle SP, Camara M, Williams P, 2000. The Pseudomonas aeruginosa lectins PA-IL and PA-IIL are controlled by quorum sensing and by RpoS. J Bacteriol 182 (22): 6401-6411. 